\title{
Multiple correspondence analysis as a tool for analysis of large health surveys in
}

African settings

Dawit Ayele, Temesgen Zewotir, Henry Mwambi

School of Mathematics, Statistics and Computer Science, University of KwaZulu-Natal, Pietermaritzburg, Private Bag X01, Scottsville 3209, South Africa.

\section{Abstract}

Background: More than two thirds of the total population of Ethiopia is estimated to be at risk of malaria. Therefore, malaria is the leading public health problem in Ethiopia.

Objective: To investigate the determinants of malaria Rapid Diagnosis Test (RDT) result and the association between socio-economic, demographic and geographic factors.

Method: The study used data from household cluster malaria survey which was conducted from December 2006 to January Method: The study une 2006 to january

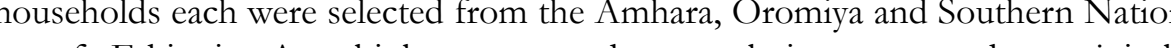
Nationalities and People (SNNP) regions of Ethiopia. A multiple correspondence analysis was used to jointly
analyse malaria RDT result, socio-economic, demographic and geographic factors.

Results: The result from multiple correspondence analysis shows that there is association between malaria RDT result and different socio-economic, demographic and geographic variables.

Conclusion: There is an indication that some socio-economic, demographic and geographic factors have joint effects It is important to confirm the association between socio-economic, demographic and geographic factors using advanced statistical techniques.

Keywords: MCA, CA, malaria, RDT.

DOI: http://dx.doi.org/10.4314/ahs.v14i4.35

\section{Introduction} While malaria has long been a cause of human suffering hara, Oromiya and Southern Nations Nationalities and and mortality in Sub-Saharan Africa, in Ethiopia the People regions of Ethiopia.

problem is particularly severe ${ }^{1}$

Malaria is a leading cause of death amongst chil- Methods and materials

dren in many African countries ${ }^{2}$ In Ethiopia malaria is a major public health problem with $68 \%$ of the total population living in areas at risk of malaria ${ }^{3,4}$. From the total population of Ethiopia, more than 50 million people are at risk ${ }^{5,6}$. In highlands or highland fridge areas of Ethiopia, i.e. mainly areas 1,000-2,000 meters above sea level ${ }^{7,8}$, epidemics of malaria are relatively high ${ }^{6,9,10}$

\section{Corresponding author:}

Ayele Dawit

University of KwaZulu-Natal, Pietermaritzburg

Private Bag X01, Scottsville 3209, South Africa.

Phone Number: +270739720957

Fax Number: +27332605648

Postal Address: School of Mathematics,

Statistics and Computer Science,

E-mail: ejigmul@yahoo.com, ayele@ukzn.ac.za
From the three regions, 5,708 households were included in the survey. From 5,708 households, Amhara, Oromiya and SNNP regions cover 4,101 (71.85\%), $809(14.17 \%)$ and 798 (13.98\%) households respectively. To conduct the survey, first, 224 Kebeles were selected. From each Kebele, 12 households were selected for malaria tests. In the survey each room in the house was listed separately. Using the presence of mosquito nets, it was possible to ascertain the density of occupation per room as well as how many sleeping rooms were in or outside each house. In addition to the number of rooms and number of nets, the persons sleeping under each net were listed. The detailed sampling procedure for the base line household survey was discussed by different authors ${ }^{11-13}$.

To obtain malaria parasite testing, consent from participants was obtained. To collect the blood sample, fingerprick blood samples was collected from participants for malaria Rapid Diagnostic Test. The test used was ParaScreen which is capable of detecting both Plasmodium falciparum and other Plasmodium species. Participants with positive rapid tests were immediately offered treatment according to national guidelines ${ }^{14,15}$.

The socio-economic, demographic and geographic covariates comprised the baseline socio-economic status,demographic, and geographic variables that included gender, age, family size, region, altitude, main source of drinking water, time taken to collect water toilet facilities, availability of electricity, radio and television, total number of rooms, main material of the room's wall, main material of the room's roof and main material of the room's floor. Malaria test RDT result, age and sex were collected at individual level. Altitude, main source of drinking water, time taken to collect water, toilet facilities, availability of electricity, radio, television, total number of rooms, main material of the room's walls, main material of the room's roof and main material of the room's floor were all collected at household level.

\section{Statistical Methods}

The cross-tabulation of categorical data is perhaps the most commonly encountered and simple form of analysis in research. Therefore, ordering things in time has been the interest of many researchers. Correspondence analysis is one of a wide range of alternative ways of handling and representing the relationships between categorical data. Correspondence analysis can ggest unexpected dimensions and relationships in the tradition of exploratory data analysis. The result of the correspondence analysis can be seen analytically and visually. This method first developed in France ${ }^{16}$ Different authors have proposed this method under various names. These methods are Dutch Homeneity Analysis ${ }^{18}$, the Japanese Qualification Method ${ }^{19}$, the Canadian Dual Scaling ${ }^{20}$. These methods have different theoretical foundations but all methods leads to equivalent solutions ${ }^{21,22}$. Correspondence analysis is thought of as principal component method for normal and contingency table data. It can be used to analyze cases-by-variable-categories matrices of non-negative data. Correspondence analysis is also a multivariate descriptive data analytic technique.

Even the most commonly used statistics for simplification of data may not be adequate for description or understanding of the data. The correspondence analysis results provide information which is similar to that produced by principal components or factor analy$\operatorname{sis}^{23}$. Using the result, it is possible to explore the structure of the categorical variables included in the table. The simplified form data provides useful information about the data ${ }^{24,25}$. The relationship of the categories of rows and columns of the data can be represented using correspondence analysis. The graphical representation of the relationships between the row and column categories is in the same space which is also produced using correspondence analysis. In general, correspondence analysis simplifies complex data and provides a detailed description of practically every bit of information in the data, yielding a simple, yet exhaustive analysis 21,26 .

Correspondence analysis has several features that distinguish it from other techniques of data analysis. The multivariate treatment of the data through multiple categorical variables is an important feature of correspondence analysis. This multivariate nature has advantage to reveal relationships which could occu during a series of pair wise comparisons of variables ${ }^{27}$. Correspondence analysis works effectively for the large data matrix, if the variables are homogeneous, and the data matrix structure is either unknown or poorly understood. There are some advantages of correspondence analysis over other methods. This advantage is related to joint graphical displays. This graphical display produces two dual displays whose row and column geometries have similar interpretations. This facilitates the analysis to detect different relationships. 

In other multivariate approaches to graphical data rep- fined by $\boldsymbol{X}=\boldsymbol{D}_{r}^{-1 / 2} \widetilde{\boldsymbol{F}} \boldsymbol{V}$
resentation, this duality is not present ${ }^{28}$.

Multiple correspondence analysis (MCA) which is where $\widetilde{\boldsymbol{F}}=\boldsymbol{D}_{r}^{-1 / 2}\left(\boldsymbol{F}-\boldsymbol{r} c^{t}\right) \boldsymbol{D}_{c}^{-1 / 2}$ and $\boldsymbol{V}_{k}$ is the $\mathrm{q} \times \mathrm{k}$ part of a family of descriptive methods, is an exten- matrix of eigenvectors corresponding to the $\mathrm{k}$ largest sion of correspondence analysis $(\mathrm{CA})$ and allows to eigenvalues $\lambda_{1}, \ldots, \lambda_{k}$ of the matrix $\widetilde{\boldsymbol{F}} \widetilde{\boldsymbol{F}}$. The investigate the pattern of relationships of several cat- projected row profiles can be plotted in the different egorical dependent variables. It is the multivariate ex- planes defined by these principal axes called row printension of $\mathrm{CA}$ to analyze tables containing three or cipal planes ${ }^{21}$

more variables. In addition to this, MCA can be considered as a generalization of principal component analysis for categorical variables which reveal patterning in complex data sets.

MCA helps to describe patterns of relationships distinctively using geometrical methods by locating each variable/unit of analysis as a point in a low-dimensional space. MCA is useful to map both variables and individuals, so allowing the construction of complex visual maps whose structuring can be interpreted. Moreover, this technique offers the potential of linking both variable centred and case centred approaches.

Suppose there are $\boldsymbol{n}$ observations on $\boldsymbol{p}$ categorical variables. Assume $\boldsymbol{q}_{j}$ different values for variable $\boldsymbol{i}$. Next define a matrix, $\boldsymbol{G}_{j}$ which is $n \times q_{j}$ matrix. This matrix is known as indicator matrix. The $n \times q_{j}$ matrix $\mathbf{G}$, with 17 . In general, $\mathrm{MCA}$ is defined as the applicathe $\boldsymbol{\sigma}_{j}$ 's 17 . In gen to the indicator matrix $\boldsymbol{G}^{29}$. Furthermore, $\boldsymbol{G}$ is divided by to the indicator matrix $\boldsymbol{G}^{2}$. Furthermore, $\boldsymbol{G}$ is divided by $\boldsymbol{F}=\frac{\mathbf{1}}{G} \boldsymbol{G}$, i.e. $\mathbf{l}_{\pi}^{\mathrm{t}} \boldsymbol{F} \mathbf{1}_{\mathrm{q}}=1$ where is $\mathbf{1}_{\mathrm{I}}$ an $i \times 1_{\mathrm{vector}}$ of ones. The vectors $\boldsymbol{r}=\boldsymbol{F} \mathbf{1}_{\boldsymbol{q}}$ and $\boldsymbol{c}=\boldsymbol{F}^{\boldsymbol{t}} \mathbf{1}_{\text {ra }}$ are the row and column marginals respectively. These marginals are the vectors of row and column masses. Suppose the diagonal matrices of the masses are defined as $\boldsymbol{D}_{r}=\operatorname{diag}(\boldsymbol{r})$ and $\boldsymbol{D}_{\mathrm{c}}=\operatorname{ding}(\boldsymbol{c})$. Note that, the $i^{\text {th }}$ element of $\mathrm{r}$ is $f=-1$ and the $\mathrm{s}^{\text {th }}$ element of $c$ is $f_{s}=\frac{w j}{n g}$ where $n$, is the frequency of category $s^{21}$.

MCA can be defined as the application of PCA to the centered matrix $\boldsymbol{D}_{r}^{-1}\left(\boldsymbol{F}-\boldsymbol{r} \boldsymbol{c}^{5}\right)$ with distances between The $\mathrm{n}$ projected coordinate of the row profiles on the principal axes are called row principal coordinates. The

$n \times k$ matrix $\boldsymbol{X}$ of row principal coordinates is de-

The categories for column profile can be described by the column profiles. The value can be calculated by dividing the columns of $\mathbf{F}$ by their column marginals. Interchanging rows with columns and all associated entities can be used for the dual analysis of columns profiles. This is done by transposing the matrix $\mathbf{F}$ and repeating all the steps. The metrics used to define the principal axes (weighted PCA) of the centered profiles matrix $\boldsymbol{D}_{r}^{-1 / 2}\left(\boldsymbol{F}-\boldsymbol{r} \boldsymbol{c}^{t}\right)^{t}$ are $\boldsymbol{D}^{c}$ and $\boldsymbol{D}_{r}^{-1}$

The $\mathrm{q} \times \mathrm{k}$ matrix $\mathrm{Y}$ of columns principal coordinates is now defined by

\section{$\boldsymbol{Y}=\boldsymbol{D}_{c}^{-1 / 2} \widetilde{\boldsymbol{F}}^{t} \boldsymbol{U}_{k}$,}

where $U_{k}$ is the $\mathrm{n} \times \mathrm{k}$ matrix of eigenvectors corresponding to the $k$ largest eigenvalues $\lambda_{1}, \ldots, \lambda_{k}$ of the matrix $\widetilde{\boldsymbol{F}} \widetilde{\boldsymbol{F}}^{t}$. To aid visualization and interpretation of the projected column profiles in the planes defined by principal axes, which are called column principal planes, can be plotted ${ }^{26}$

The absolute contribution of the variable $j$ to the inertia of the column principal component $\alpha$ in the $\alpha^{\wedge}$ th column of $\mathrm{Y}$ is given by

$$
c_{j \alpha}=\sum_{s \in M_{j}} s \in M_{j} f_{. s} y_{s \alpha}^{2}
$$

where $M_{j}$ is the set of categories of variable $j$. The relation between the absolute contribution $c_{j \alpha}$ and the correlation ratio between the variable $j$ and the row standard component is given by

$$
\eta_{j \alpha}^{2}=\sum_{s \in M_{j}} \frac{n_{s}}{n}\left(\bar{x}^{*}-D\right)^{2}=p \times c_{j \alpha},
$$

Note that factor loadings for PCA are correlations between the variables and the components (the correlation ratios) are known as discrimination measures. More details for correspondence analysis can be found in different literatures ${ }^{21,23-27}$.
The application of multiple correspondence analysis is useful to visualize the associations between the socioeconomic, demographic and geographic parameters and the malaria RDT result. Therefore, applying correspondence analysis helps to reduce the interaction parameters. Furthermore, the graphical interpretation of the data could be useful tool in an exploratory research and the reduction of the level of the associations between the investigated parameters.
For the applications of MCA, variables were divided into different subgroups that contain variables of simigeographic variables. Variables analyzed with MCA generally are assumed to be categorical. This technique is described by Guitonneau and Roux ${ }^{30}$. To apply MCA to both continuous and discrete data, continuous variables could be categorized through a process of mutually exclusive and exhaustive discretization or coding ${ }^{17}$. Multiple correspondence analysis locates all the categories in a Euclidean space. To examine the associations among the categories, it is important to plot the first two dimensions of the Euclidean space. For the multiple correspondence analysis, malaria RDT result graphic variables were considered. The demographic variables are sex, age and family size. For the multiple correspondence analysis, the continuous age and family size variables were recorded to be appropriate for the analysis.

The socio- economic variables are source of drinking water, time to collect water, toilet facility, availability of radio, television and telephone, construction material for room's floor, wall and roof, use of anti-mosquito spray, use of mosquito nets, total number of rooms in the house and total number of nets in the house. Besides the socio-economic and demographic variable, there were geographic variables included in the analysis. These variables are region and altitude. To be lar types such as socio-economic, demographic and and the other socio-economic, demographic and geo- ppropriate to MCA analysis, altitude has been recoded a categorical variable. Therefore, to perform the MCA analysis all socio- economic, demographic and geographic variables were included to the multiple correspondence analysis. The MCA analysis was performed using SAS 9.3 software.

In the MCA analysis, each principal inertia values expressed as a percentage of the total inertia. These values quantify the amount of variation accounted for by the corresponding principal dimension. In addition to this the principal inertia is decomposed into components for each of the rows and columns. The decomposed rows and columns provide the numerical contributions used to interpret the dimensions and the quality of display of each point in the reduced space. The parts which expressed as percentages are useful to explain the method of determination of the dimensions. The same parts of the dimensions can be expressed relative to the inertia of the corresponding points in the full space and this help to assess how close the individual points are to the dimension.

Table 1 presents inertia and Chi-Square decomposition for multiple correspondence analysis. Correspondence analysis employs chi-square distances to calculate the dissimilarity between the frequencies in each cell of a contingency table. The calculation of the chi-square distances is cell- independent. Pairs of cells whose observed and expected values are the same and can be considered to be independent of each other. Therefore, pairs of cells for observed and expected values are different. Table 1 suggested that the dimensions 1 and 2 account for $19.4 \%$ of the total association. The total chi-square statistic in Table 1, which is a measure of the association between the rows and columns in the full dimensions of the table, is 2,169,476 with degrees of freedom 2050. This chi-square represents all pairwise interactions among the factors. The maximum number of dimensions (or axes) is the minimum of the number of rows and columns, minus one. 
Table 1: Inertia and Chi-Square Decomposition

\begin{tabular}{|c|c|c|c|c|c|}
\hline $\begin{array}{l}\text { Singular } \\
\text { Value }\end{array}$ & $\begin{array}{l}\text { Principal } \\
\text { Inertia }\end{array}$ & $\begin{array}{l}\text { Chi- } \\
\text { Squar }\end{array}$ & Percent & $\begin{array}{l}\text { Cumulative } \\
\text { Percent }\end{array}$ & $\begin{array}{lllll}2 & 4 & 6 & 8 & 10 \\
- & -\end{array}$ \\
\hline 0.42757 & 0.18282 & 232503 & 10.72 & 10.72 & $* * * * * * * * * * * * * * * * * * * * * * * * * * * * *$ \\
\hline 0.38438 & 0.14775 & 187901 & 8.66 & 19.38 & 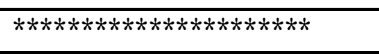 \\
\hline 0.3126 & 0.09772 & 124277 & 5.73 & 25.11 & $* * * * * * * * * * * * * * * *$ \\
\hline 0.29555 & 0.08735 & 111089 & 5.12 & 30.23 & $* * * * * * * * * * * * * * *$ \\
\hline 0.28047 & 0.07866 & 100043 & 4.61 & 34.84 & $* * * * * * * * * * * * *$ \\
\hline 0.26462 & 0.07002 & 89054 & 4.1 & 38.94 & $* * * * * * * * * * * *$ \\
\hline 0.26193 & 0.06861 & 87250 & 4.02 & 42.97 & $* * * * * * * * * * * * *$ \\
\hline 0.25503 & 0.06504 & 82716 & 3.81 & 46.78 & $* * * * * * * * * * * *$ \\
\hline 0.24806 & 0.06154 & 78259 & 3.61 & 50.39 & $* * * * * * * * * *$ \\
\hline 0.24591 & 0.06047 & 76909 & 3.55 & 53.93 & $* * * * * * * * * *$ \\
\hline 0.24557 & 0.06031 & 76696 & 3.54 & 57.47 & $* * * * * * * * * *$ \\
\hline 0.24356 & 0.05932 & 75444 & 3.48 & 60.94 & $* * * * * * * * * *$ \\
\hline 0.23959 & 0.0574 & 73005 & 3.37 & 64.31 & $* * * * * * * * *$ \\
\hline 0.23772 & 0.05651 & 71869 & 3.31 & 67.62 & $* * * * * * * *$ \\
\hline 0.23474 & 0.0551 & 70079 & 3.23 & 70.85 & $* * * * * * * *$ \\
\hline 0.23154 & 0.05361 & 68179 & 3.14 & 73.99 & $* * * * * * * *$ \\
\hline 0.22675 & 0.05142 & 65388 & 3.01 & 77.01 & $* * * * * * * *$ \\
\hline 0.22274 & 0.04961 & 63094 & 2.91 & 79.92 & $* * * * * * * *$ \\
\hline 0.21997 & 0.04839 & 61539 & 2.84 & 82.75 & $* * * * * * * *$ \\
\hline 0.21788 & 0.04747 & 60370 & 2.78 & 85.54 & $* * * * * * * *$ \\
\hline 0.2095 & 0.04389 & 55817 & 2.57 & 88.11 & $* * * * * *$ \\
\hline 0.2031 & 0.04125 & 52458 & 2.42 & 90.53 & $* * * * * *$ \\
\hline 0.1965 & 0.03861 & 49106 & 2.26 & 92.79 & $* * * * * *$ \\
\hline 0.18357 & 0.0337 & 42856 & 1.98 & 94.76 & $* * * * *$ \\
\hline 0.17417 & 0.03033 & 38578 & 1.78 & 96.54 & $* * * *$ \\
\hline 0.16618 & 0.02761 & 35119 & 1.62 & 98.16 & $* * * *$ \\
\hline 0.14744 & 0.02174 & 27646 & 1.27 & 99.44 & $* * *$ \\
\hline 0.08754 & 0.00766 & 9745 & 0.45 & 99.89 & * \\
\hline 0.04423 & 0.00196 & 2488 & 0.11 & 100 & \\
\hline Total & 1.70588 & 2169476 & 100 & & \\
\hline
\end{tabular}

From Table 1, the singular value indicates the relative the analysis ${ }^{25}$. However, the proportion of variance contribution of each dimension to an explanation of explained by each dimension must be balanced with the inertia, or proportion of variation, in the participant the cut-off point. The singular value and the inertia are and variable profiles. The singular values can be inter- directly related i.e., the inertia is an indicator of how preted as the correlation between the rows and columns much of the variation in the original data is retained in of the contingency table. As in principal components the dimensional solution ${ }^{31}$. Furthermore, the percentanalysis, the first dimension explains as much variance ages of inertia accounted for by the first twelve axes as possible, the second dimension is orthogonal to the are 10.7 per cent and 8.7 per cent, 5.73 per cent, 5.12 first and displays as much of the remaining variance per cent, 4.61 per cent, 4.1 per cent, 4.02 per cent, 3.81 as possible, and so on. Singular values of greater than per cent, 3.61 per cent, 3.55 per cent, 3.54 per cent and 0.2 indicate that the dimension should be included in 3.45 per cent, respectively (Table 1).
Based on this result, the first twelve axes as accounting method to assess most appropriate number of dimenfor similar amounts of variance and would expect 39.1 sions for interpretation is using scree plot. The scree per cent of the inertia to be accounted for by the re- plot presents the proportions of variance explained ${ }^{25}$. maining axes. As can be seen from the table, 93 per cent As can be seen from the figure, the scree plot suggests of the association can be represented well in twenty that the proportion of variance explained drops faster three dimensions. However, these data can be consid- up to 7 th dimension and less rapidly up to dimension ered in just two dimensions. The first axis account- ${ }^{26}$. As discussed by $\mathrm{Hair}^{25}, 0.2$ can be considered as a ing for approximately 10.72 per cent of the inertia and cut-off point as a fir the second axis accounts approximately 8.66 per cent. gests that only 90.5 per cent variation can be exThe percentages of inertia in MCA are low and tend to plained by 22 dimensions. However, working 22 dibe close to one another and this latter fact might lead to mensions would not achieve the conceptual clarity for an assumption that individual axes might be unstable. the use of correspondence analysis. But interpreting 22 dimensions is ur Figure 1 presents the scree plot of singular values. One sional scaling solutions, usually two or three dimensions are interpreted.

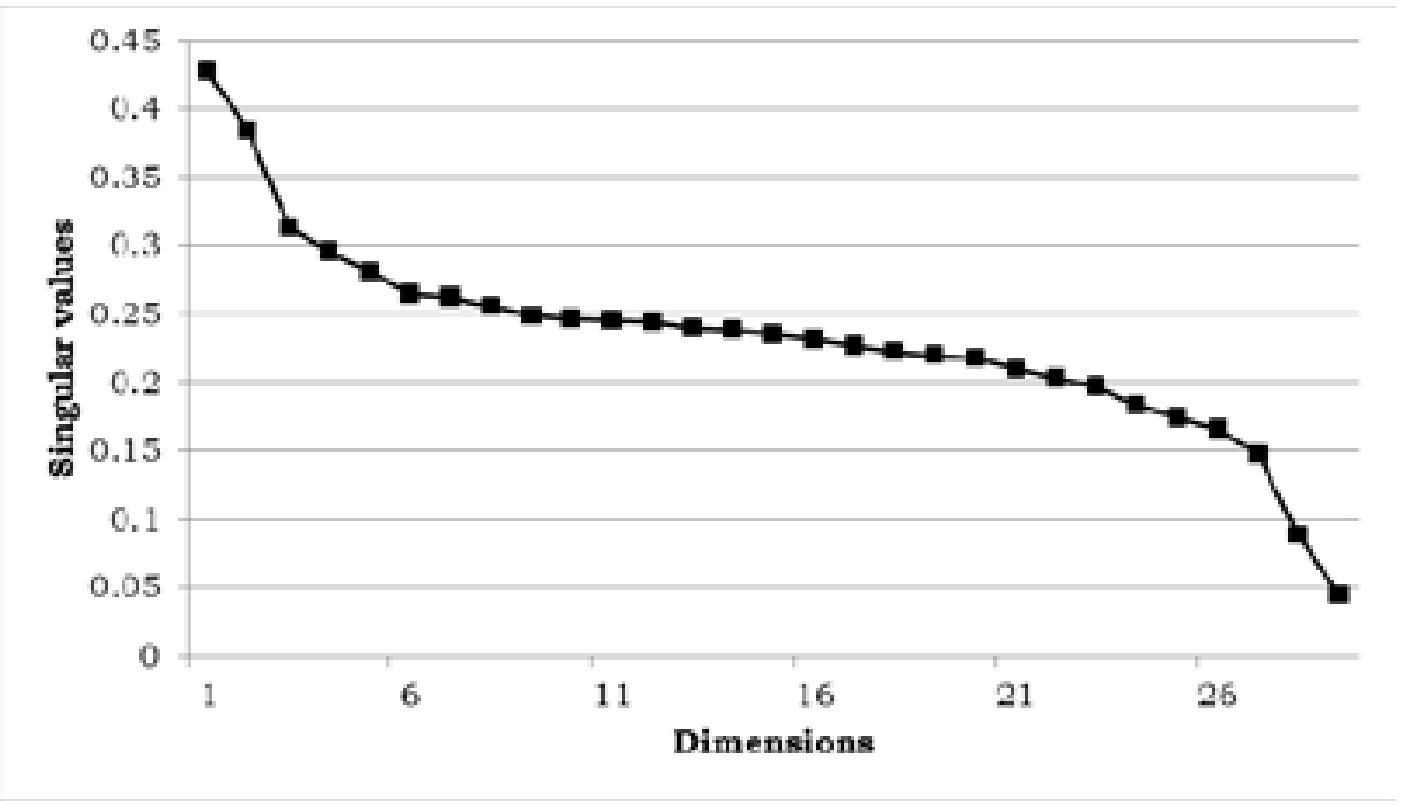

Figure 1: Scree plot of singular values

Figures $2 \mathrm{a}$ and $2 \mathrm{~b}$ contain the multiple correspond- ables for twelve dimensions, with Dimension 1 on the ence analysis scaling solution coordinates for the vari- horizontal axis and Dimension 2 on the vertical axis and so on. 
Figure 2a: Multiple correspondence analysis plot for dimensions 1 and 2

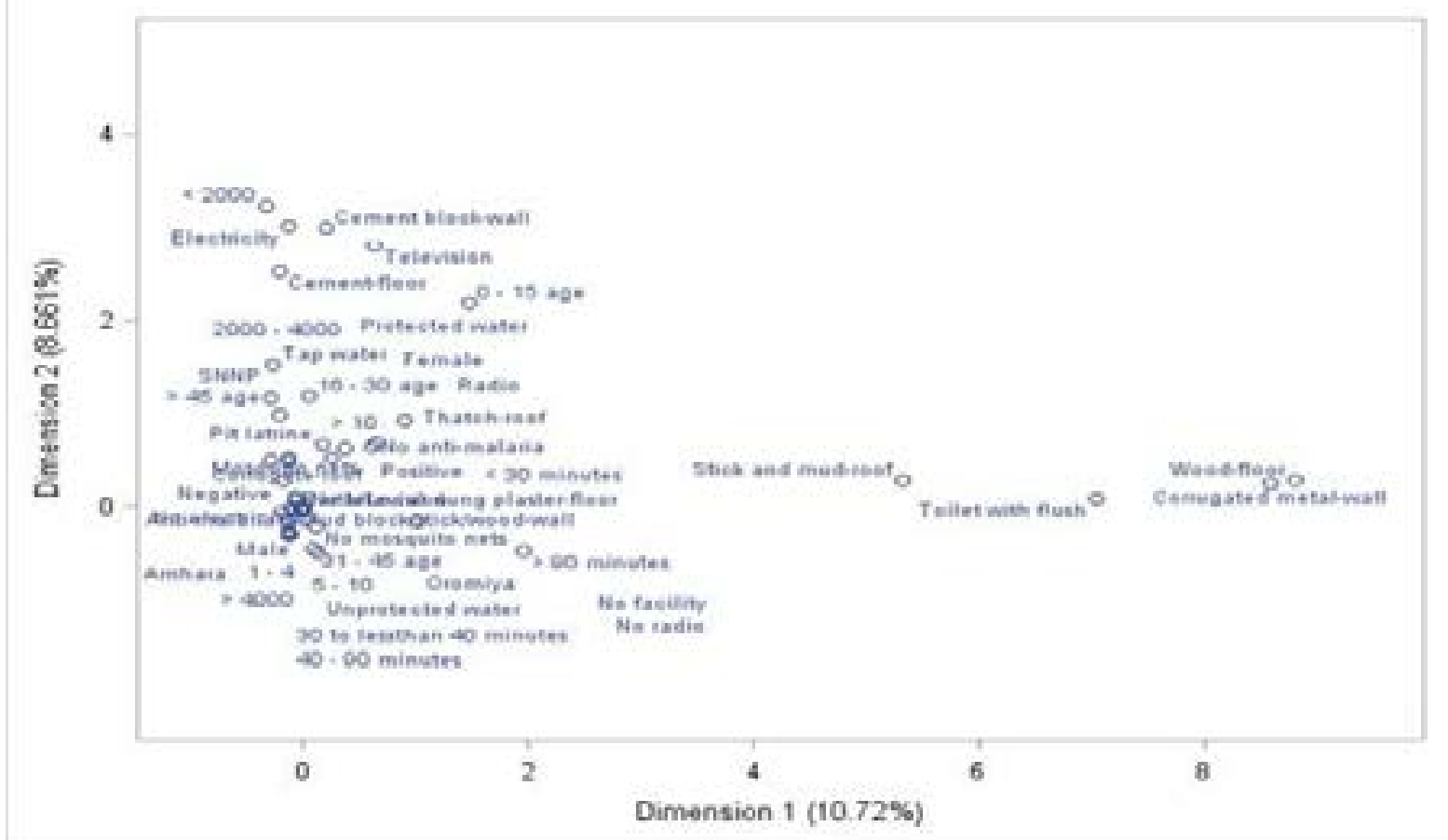

Multiple correspondence analysis locates all the cat- stick and mud roof, toilet with flush, wood floor and egories in a Euclidean space. The first two dimensions corrugated metal wall appears separately in the right of this space are plotted to examine the associations hand side of the chart. Therefore, these variables have among the categories. Dimension 1 accounts for to be included in the interpretation of dimension 1 and 10.72 per cent of the variance in the data and Dimen- similarly for other dimensions.

sion 2 accounts for 8.66 per cent of the variance (Figure

2a). The twelve dimensions totally accounts for 60.9 per It is important to note that this two-dimensional chart is cent of the variations. It can be seen that variable like part of the twenty two dimensional solutions. Interpret-

\section{Figure 2b: Multiple correspondence analysis plot for eleven dimensions}
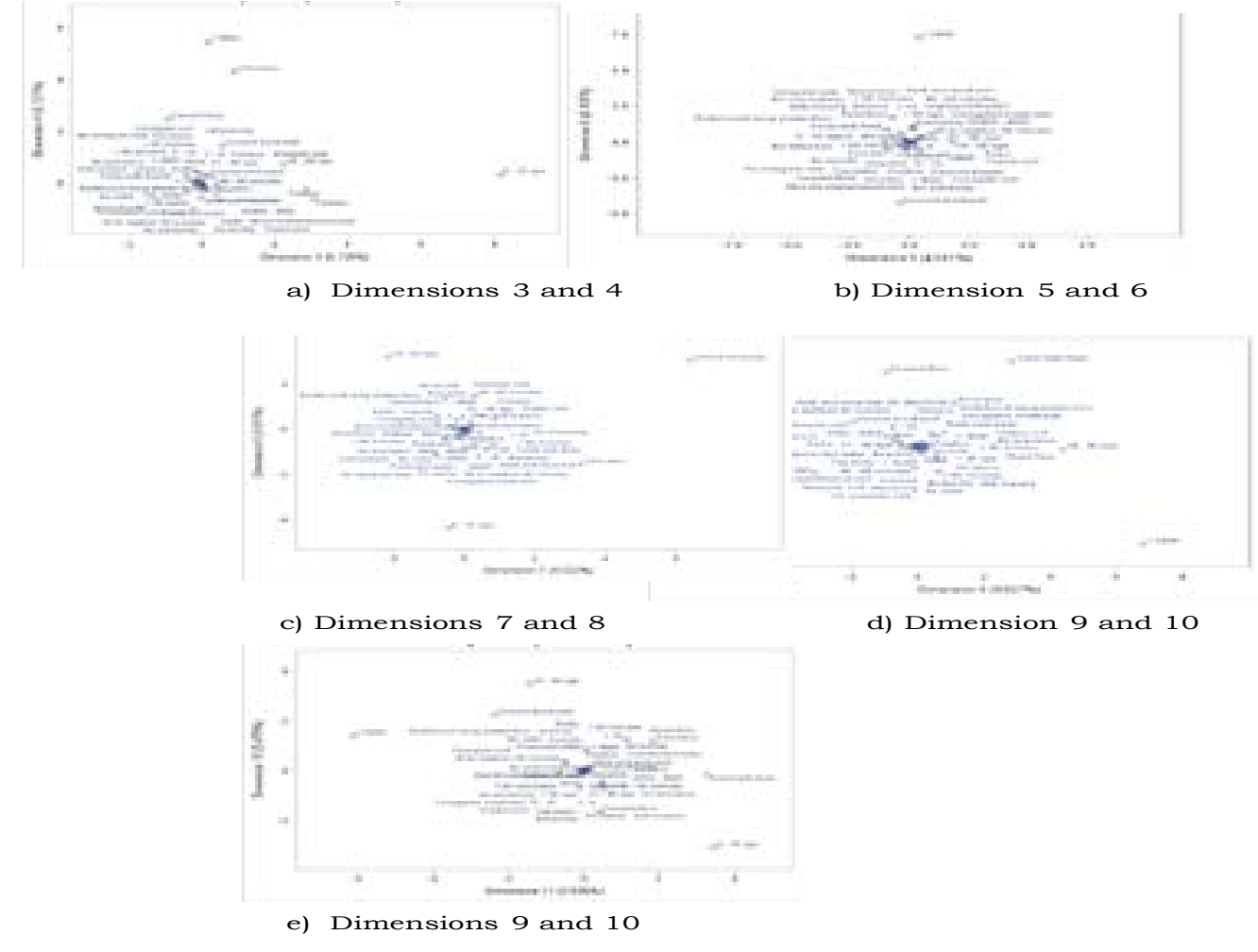

d) Dimension 9 and 10 ing of each dimension is considered as the contribution of variables to that dimension ${ }^{32}$. This is because a variable that appears on the two-dimensional chart might be a major contributor to another dimension but might not be located in the existing two-dimensional plane $^{33}$. As can be seen in Figure 2a, the right quadrant of the plot (dimensions 1 and 2) shows that the categories stick and mud roof, toilet with flush, wood floor and corrugated metal wall are associated. To the top of the plot,altitude less than 2000 meter, use of electricity, cement block wall, cement floor, use of television, protected water, altitude between 2000-4000 meters are associated. On the other hand, positive malaria RDT result, not using anti-mosquito spray, thatch roof, earth or dung plaster floor are grouped together. Furthermore, negative malaria RDT result, use of anti-mosquito spray, use of malaria nets, pit latrine toilet and corrugated floor are associated. Similarly, unprotected water, 30-40 minutes walk to get water, no toilet facility and no radio are associated together. This interpretation of the plot is based on points found in approximately the same direction and in approximately the same region of the space.

So far, the association between socio-economic, demographic, geographic variables and malaria RDT result was assessed based on dimension 1 and 2. As can be seen from Table 2 a, dimension 1 and 2 constitute 19.4 per cent of the variation. But, the other 20 dimensions all together constitute 71.2 per cent of the variation. Except the relationship between dimension 4 and 3 , dimension 5 and 2 , dimension 5 and 3 and dimension 7 and 1 , the relationship between the variables for other combination of dimensions shows that it is located at the center of the graph. The relationship between variables shows similar relationship as of dimension 1 and 2 .

\section{Discussion and conclusion}

In this study, multiple correspondence analysis was used as a way to graphically represent and interpret the relations between primary meanings in different malaria RDT result, socio-economic, demographic and geographic variables. Multiple correspondence analysis provides useful interpretative tools that can further the understanding of the conceptual context in which socio-economic, demographic and geographic variables by malaria RDT result occurs.

As it was discussed above, multiple correspondence analysis is a method for exploring associations between ts of categorical variables. Mathematically, it is a method for breaking down the value of the goodnessof-fit statistic into components due to the rows and columns of the contingency table. It can also be considered as a technique for assigned order to unordered categories. Therefore, the MCA approach involves defining a set of points, with associated masses, in a multidimensional space structured by Euclidean distance. Furthermore, the display is also thought of as a framework for reconstructing the original data as closely as possible. To display the relationship, coordinate positions of the row and column points are used.

The association using MCA gives the relationship among coded variables and their associations. The technique allows the analysis of the relationships between the variables and different levels of one variable. Furthermore, the results of the analysis can be seen analytically and visually. This method of display gives detailed information of the relationship between variables and their associations. Therefore, the result from multiple correspondence analysis shows that there is association between malaria RDT result and different socio-economic, demographic and geographic variables. Moreover, there is an indication that some socioeconomic, demographic and geographic factors have joint effects.

It is important to confirm the association between socio-economic, demographic and geographic factors using advanced statistical techniques. Therefore, future investigations need to be done to identify those variables that show significant relationships. By identifying those variables which could have joint effect, it is important to determine the principal axes and the identification of selection of variables to take forward for further analysis. In conclusion, the aim of the multiple correspondence analyses was to summarise the multidimensional data into an interpretable smaller dimensional factor and to reveal some association between different types of respondents. But, this reduction was not suitably achieved. This can be put down to either (i) all the factors being too scattered to be summarized in a smaller dimension, and/or (ii) the number of observations obtained in the cross tabulation being too small for all possible pairs of levels in the study.

\section{Acknowledgements}

We thank, with deep appreciation, The Carter Center of Ethiopia, for providing and giving permission to use the data for this study. 


\section{Ethical clearance}

9. Adhanom, T., et al., Malaria. In The Eipdemiology . Emory University Institutional Review Board (IRB tion, in Edited by: Berhane Y, Hailemariam D, Kloos H 1816) and Amhara, Oromiya and SNNP regional health and Shama PLC. Addis Ababa, Ethiopia 2006. p. 556bureaux. Informed consent was sought in accordance 576.

with the tenets of the declaration of Helsinki.

\section{Competing interests}

The authors declare that they have no competing interests.

\section{Authors' contributions}

DGA acquired the data, performed the analysis and drafted the manuscript. T'TZ and HGM designed the research. All authors discussed the results and implications and commented on the manuscript at all stages. All authors contributed extensively to the work presented in this paper. All authors read and approved the final manuscript. All authors contributed extensively to the work presented in this paper.

\section{References}

1.WHO.Malaria:http://www.who.int/mediacentre/ factsheets/fs094/en/ 2012 [cited 2012 May].

2. Snow, R.W., N. Peshu, and D. Forster, Environmental and entomological risk factors for the developmen of clinical malaria among children on the Kenyan coast. Transactions of the royal society of tropical medicine and hygiene, 1998. 92: p. 381 - 385.

3. Adugna, A., Malaria in Ethiopia http://www.ethiodemographyandhealth.org/ MedVectoredDiseasesMalaria.pdf. Accessed Nov. 14, 20112011, Addis Ababa, Ethiopia: Ethiopian Demography and Health.

4. Federal Ministry of Health (FMH), Malaria and Other Vector-borne DiseasesControl Unit, 1999: Addis Ababa, Ethiopia.

5. FMH, Federal Ministry of Health: Malaria: Diagnosis and Treatment Guidelines for Health Workers in Ethiopia. Addis Ababa, Ethiopia, Federal democratic Republic of Ethiopia, Ministry of Health, 2004.

6. FMH, National five-year strategic plan for malaria prevention and control in Ethiopia 2006 - 2010, 2006. 7. WHO, World Health Organization: Systems for the early detection of malaria epidemics in Africa: an analysis of current practices and future priorities, country experience. Geneva, Switzerland, World Health Organization, 2006.

8. Zhou, G., et al., Association between climate variability and malaria epidemics in the East African highlands. Proceedings of the National Academy of Sciences 2004. 101: p. 2375-2380. Kloos $\mathrm{H}$ and Zein AZ. Boulder, USA, Westview Press Inc.1993: p. 341-352.

11. Ayele, D.G., T. Zewotir, and H. Mwambi, Prevalence and risk factors of malaria in Ethiopia. Malaria Journal, 2012. 11:195 p. doi:10.1186/1475-2875-11-

Emerson, P.M., et al., Integrating an NTD with One of "The Big Three": Combined Malaria and Trachoma Survey in Amhara Region of Ethiopia. PloS Neglected tropical diseases, 2008. 2(8).

13. Shargie, E.B., et al., Malaria prevalence and mosquito net coverage in Oromia and SNNP regions of Ethiopia. BMC Public Health, 2008. 8:321.

14. WHO, Malaria Rapid Diagnostic Test Performance, 2009: Geneva, Switzerland.

15. Wongsrichanalai, C., et al., A Review of Malari 15. Wiagnostic Tools: Microscopy and Rapid Dingnosic Test (RDT). The American Journal of Tropical Medicine and Hygiene, 2007. 77 (Suppl 6): p. 119-127.

16. Benzécri, J.-P., Analyse des Donnees Vols 1 and 2 1973 Dunod.

17. Greenacre, M.J., Theory and Applications of Correspondence Analysis 1984: Academic Press.

18. Gifi, A., Nonlinear Multivariate Analysis1990, New York: John Wiley \& Sons.

19. Hayashi, C., Multidimensional quantication \{ with plications to analysis of social phenomena. Annals of the Institute of Statistical Mathematics, 1954. 5(2) p. 121-143.

20. Nishisato, S., Analysis of categorical data: Dual Scaling and its applications1980, Toronto: University of Toronto Press.

21.Greenacre, M.J. and J. Blasius, Multiple correspondence analysis and related methods 2006, Boca Raton: Chapman \& Hall/CRC synthesis of multiple correspondence analysis, optima scaling, dual scaling homogeneity analysis and other methods for quantifying categorical multivariate data. Psychometrika, 1985. 50: p. 91-119.

23. Hill, M.O., Correspondence Analysis: A Neglected 1974. 23 (3): p. 340-354

24. Van der Heijden, P.G.M. and J. de Leeuw, Corre- spondence analysis used complementary to loglinear 29. Benzécri, J.P., Analyse des Donnkes, analysis. Psychometrika, 1985. 50 (4) p. 429-447. 25. Hair, J.F., et al., Multivariate Data Analysis. Vol. 4. Dunod.

1995, Upper Saddle River, NJ: Prentice Hall.

30. Guitonneau, G.G. and 26. Johnson, R.A. and D.W. Wichern, Applied multi- du Genre Erodium. Cahiers de l'Analyse des Donnees, variate statistical analysis.Vol. 6. 2007, New Jersey: Pear- 1977.2 (1): p. 97-113.

and Tulu, N.A., Malaria. In The Ecology of Healt

22. Tenenhaus, M. and F.W. Young, An analysis and Multivariate Method. Journal of the Royal Statistical Society,
son/Prentice Hall.

31. Bendixen, M. A practical guide to the use respondence Analysis with MATLAB. Computers \& Marketing Research On-Line, 2003, 16-38. http://mro. Geosciences 1993. 19 (7): p. 1007-1022. 28. Askell-Williams, H. and M.J. Lawson, A Cor- 32. Clausen, S.-E., Applied Correspondence Analrespondence Analysis of Child-Care Students' and ysis. An Introduction1998, Thousand Oaks, CA: Sage. Medical Students' Knowledge about Teaching and 33. Nishisato, S., Elements of dual scaling: An Learning. International Education Journal, 2004. 5 (2). introduction to practical data analysis 1994, Hillsdale, $\mathrm{NJ}$ : Erlbaum 